\title{
ANÁLISE DA QUALIDADE DE ENERGIA ELÉTRICA NA INDÚSTRIA
}

\author{
Gabriel Mendonça Guimaraes - gabriel.guimaraes@alunos.unis.edu.br \\ Paulo Antônio Souza - Paulo.nunes@alunos.unis.edu.br \\ Eric de Cassio - eric.mendonca@alunos.unis.edu.br \\ Tiago Bittencourt Nazaré - tiago@unis.edu.br
}

RESUMO:Através de uma pesquisa e trabalho de campo, buscou-se descrever os fatores que influenciam na condição da energia recebida por uma determinada fábrica, discernir a tensão de boade má qualidade, demonstrar por gráficos obtidos através de uma análise técnica, os valores a serem examinados para que se chegue à conclusão de que o circuito que foi analisado estava sendo prejudicado ou não pela energia recebida.

Na linha de produção da indústria onde foi realizada a análise, vinham ocorrendo oscilações na energia recebida da concessionária e isso acabou afetando a produtividade da mesma, pois impactou diretamente no funcionamento de algumas máquinas. Pelo fato de haverem equipamentos importados, os quais seguem as normas do país de origem, mesmo com os níveis de energia registrados estando dentro do padrão da concessionária local e dos valores determinados pela ANEEL, ainda assim, impactavam no funcionamento das máquinas. A partir desta necessidade, uma empresa especialista na área foi contratada para efetuar medições no circuito, fazer uma análise para descobrir a causa e buscar soluções para este problema com a energia recebida.

A energia elétrica se tornou um subsídio básico e fundamental no desenvolvimento da sociedade. $\mathrm{Na}$ indústria, além da presença, a qualidade deste elemento passa a ser de extrema importância tanto para eficiência quanto para a vida útil dos componentes e maquinários. Uma energia apropriada é um problema a menos a ser enfrentado por uma fábrica e seus colaboradores em sua rotina.

Palavras-Chave: Qualidade, Energia, Indústria, Análise. 


\section{INTRODUÇÃO}

O tema Qualidade da Energia, em inglês, "Power Quality", é uma expressão referente a energia elétrica que é fornecida com sua onda em forma senoidal pura, ou seja, sem alterações na sua amplitude. Este tema abrange muitos fatores onde os principais estão presentes neste artigo. Voltar a atenção para a qualidade da energia é uma consideração muito importante para toda empresa, e devido a sua importância, é fundamental que seja avaliada como qualquer recurso básico para o funcionamento e desenvolvimento de uma infraestrutura bem elaborada.

Quando equipamentos industriais começam a apresentar falhas permanentes ou mau funcionamento, é aconselhado que se faça uma análise afim de identificar se a causa destes problemas está na energia elétrica fornecida aos circuitos. Os problemas com a Qualidade da Energia Elétrica geralmente são provenientes de três fatores:

Tensões e correntes harmônicas: De acordo com Kagan, Robba, Schmidt (2009), no livro Estimação de indicadores de qualidade da energia elétrica, define-se o termo distorções harmônicas como as ondas não-senoidais de corrente e tensão provocadas por equipamentos cuja carga é nãolinear. Tais equipamentos, como um inversor de frequência, por exemplo, quando conectado à rede, contribuirá na distorção harmônica, onde o próprio equipamento também é sensível ao fenômeno.

Sendo assim, a existência da distorção harmônica em um circuito é indesejável pois, ainda no exemplo do inversor, irá reduzir a vida útil do equipamento, gerando custos de manutenção, produção e até reinvestimentos em novos equipamentos para substituição.

Figura 1 - Exemplo de carga não linear
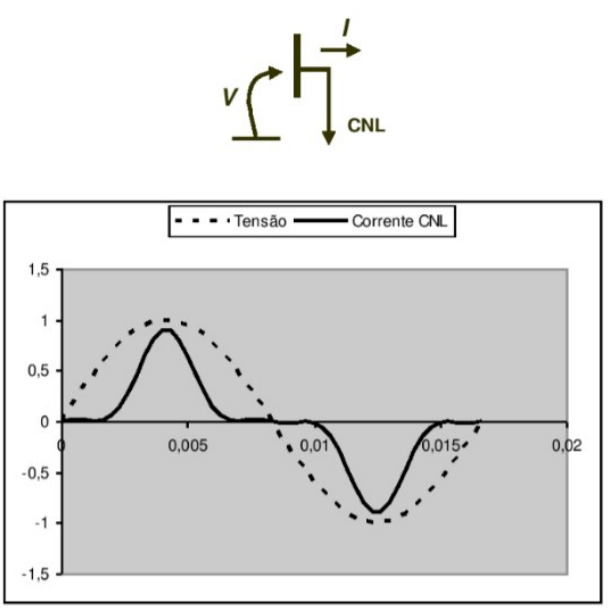

Fonte: Livro Estimação de indicadores de qualidade da energia elétrica (2009, p.196)

A figura 1 retirada do livro Estimação de indicadores de qualidade da energia elétrica, mostra um exemplo de uma carga onde foi aplicada uma tensão senoidal, porém, sendo a carga nãolinear, a resultante será uma corrente cuja onda é não-senoidal, embora ela esteja correlacionada à frequência da tensão.

Baixo fator de potência: O Fator de Potência (FP) é uma medida de quanto da potência elétrica consumida está de fato sendo convertido em trabalho útil. A ANEEL, através do módulo 8 do Prodist, o qual trata sobre a Qualidade da Energia, estabelece que o valor do fator de potência deve estar compreendido entre 0,92 e 1 indutivo ou 1 e 0,92 capacitivo. Quanto menor este valor, mais ineficiente está sendo o aproveitamento da energia, onde uma parte é convertida em trabalho e a outra, não utilizada, é chamada de energia reativa. Um valor inferior a 0,92 de fator de potência, habilita a Concessionária a cobrar multa na fatura de energia sobre o consumo de Potência Reativa 
além dos $8 \%$ máximos permitidos. Corrigir o fator de potência é fundamental em qualquer instalação industrial.

Para se calcular o valor do fator de potência a partir dos valores de potência ativa e reativa (P e Q) registrados nas medições, utiliza-se a fórmula da figura 2 a seguir:

Figura 2: Fórmula para cálculo do fator de potência

$$
f p=\frac{P}{\sqrt{P^{2}+Q^{2}}}
$$

Fonte: ANEEL, PRODIST. Módulo 8 - Qualidade da energia

Quedas de tensão, perdas, sobrecargas são algumas das consequências de um fator de potência baixo numa instalação.As principais cargas que causam baixo FP são lâmpadas fluorescentes, transformadores em vazio (sem carga) ou com baixa carga e motores de indução (motores mais usados na indústria). A forma de compensar o baixo Fator de Potência é a instalação de bancos de capacitores em paralelo na entrada de energia ou no próprio equipamento com carga indutiva. Esses bancos introduzem na instalação uma carga capacitiva, que tem o efeito contrário da carga indutiva. Isso compensa o baixo valor e o ajusta para mais próximo de 1 , evitando as multas.

Instabilidade da tensão: É uma consequência de um fornecimento instável de níveis de eletricidade. A baixa tensão causa ineficiência no circuito e obviamente, pode causar quedas de energia. A elevação na tensão, além de prejudicar o desempenho atual de um equipamento, reduz sua longevidade.

O gerenciamento da qualidade da energia, vai garantir que a tensão disponibilizada ao circuito seja estável, conforme exigem as máquinas presentes nos circuitos.

Nas páginas seguintes deste artigo, são demonstrados os resultados obtidos em uma análise de campo, onde uma empresa enfrentava problemas na qualidade da energia que recebia em sua fábrica. Foi contratada uma equipe especializada na área para que através de medições, pudessem descobrir a causa dos problemas e buscar soluções,evitando assim prejuízos com paradas repentinas na produção.Vale ressaltar, que esta mesma empresa contratante, havia tomado iniciativa de instalar um autotrafo com regulador de tensão diretamente na entrada do equipamento que sofria com as oscilações na tensão.

\section{REVISÃO DE LITERATURA}

A qualidade de energia é caracterizada basicamente pela forma de onda de tensão dos componentes de um sistema trifásico, tambémé chamada de qualidade da tensão e contempla os seguintes fenômenos: distorções harmônicas, baixo fator de potência, instabilidade da tensão dentre outros fatores. (KAGAN; ROBBA; SCHIMIDT, 2009, p.2).

Distorção Harmônica em uma rede elétrica se refere ao surgimento de correntes e tensões não senoidais por cargas não-lineares conectadas à rede. (KAGAN; ROBBA; SCHIMIDT, 2009, p.195). 
Quando cargas indutivas são acionadas com alimentação por corrente alternada, ocorre um fenômeno de defasagem entre as ondas da tensão e da corrente, causando o surgimento da Potência Reativa. Esta defasagem é quantificada pelo chamado Fator de Potência (FP). (SCARPIN, 2017) Segundo Kagan, Robba e Schimidt (2009) as variações de tensão de longa e curta duraçãoocorrem quando há distorções nos níveis de corrente e tensão geralmente causadas por faltas no sistema elétrico de abastecimento ou até mesmo por problemas ocasionados nos circuitos internos das instalações, como exemplo, motores ligados ao sistema de distribuição.

\section{METODOLOGIA}

O estudo foi feito com base nas análises da qualidade de tensão na instalação da unidade industrial de uma fábrica, em especial a alimentação de uma máquina sopradora de garrafa PET. A arquitetura do circuito onde foram feitos os testes é composta, conforme a Figura 3, de um transformador de 500kVA que alimenta um QDC em 380V. A medição foi feita na saída do quadro. No circuito existe ainda um autotransformador que se ajusta automaticamente a eventuais sobretensões permanentes na rede de alimentação por meio da utilização de comutadores de "tap's".

Figura 3: Arquitetura do Circuito obtida do relatório

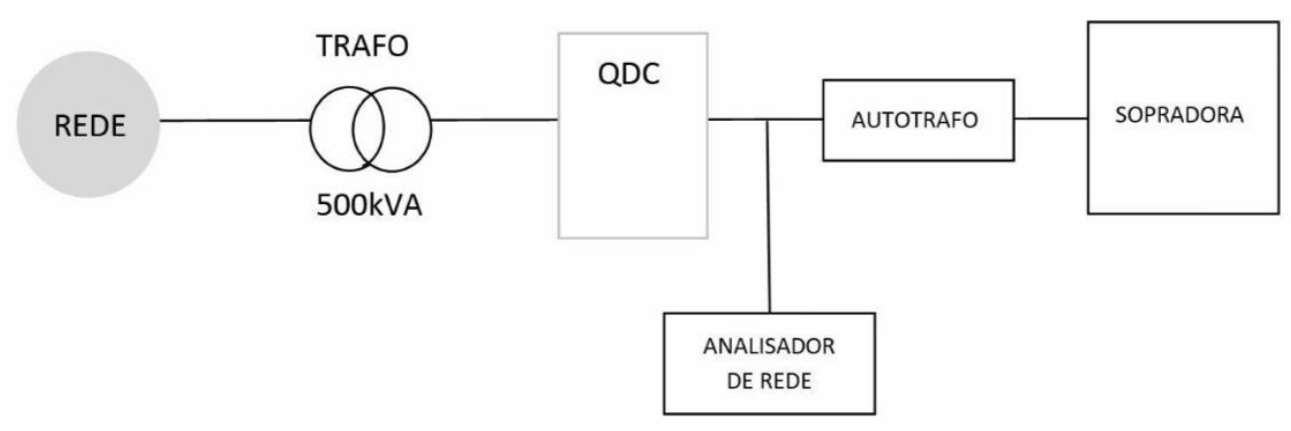

Fonte: Relatório de Qualidade da Energia executado em campo(2020)

As medições foram realizadas com o uso de um analisador de rede EMBRASUL RE4000. O arquivo de dados gerados pelo equipamento de medição apresenta grandezas como tensão, corrente, potência, fator de potência, distorções harmônicas, dentre outras. A análise dos relatórios fornecidos tem como base o principal guia vigente e referêncianacional PRODIST Módulo 8 da ANEEL.

\section{RESULTADOS OBTIDOS}


Colocando o estudo em prática, através da medição em campo nos níveis de DHTv (Distorções Harmônicas Totais de Tensão) feita em uma indústria, pode-se observar que máquinas rotativas e transformadores, em regime permanente e em condições normais de operação, não causam distorções na rede. Porém, em momentos transitórios da produção ou quando as máquinas operam fora de sua condição nominal, foi observado breves picos nos níveis de harmônica, que podem ser observados na figura 4.

Figura 4: Análise dos níveis de harmônica

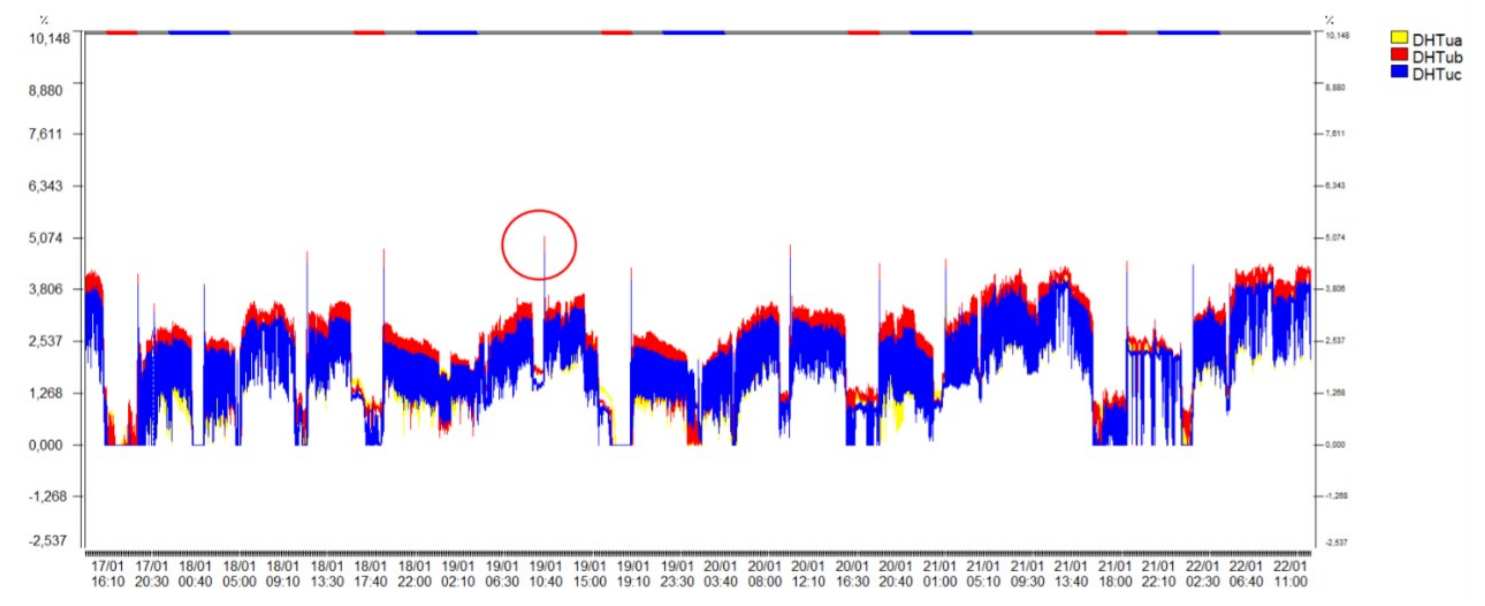

Fonte: Relatório de Qualidade da Energia executado em campo (2020)

Foi feita uma medição em cada fase do circuito. A figura 4 demonstra tais resultados obtidos em uma fase. De acordo com o módulo 8 do PRODIST da Agência Nacional de Energia Elétrica ANEEL, referente a Qualidade da Energia Elétrica, tais níveis medidos em campo estão dentro dos coeficientes aceitáveis. Onde somente níveis de distorções acima de 10\% são considerados prejudiciais em circuitos com a tensão nominal respectiva a que esta indústria recebe.

Nas medições do fator de potência, a partir das leituras realizadas, é possível observar que os valores estão adequados, pois, também de acordo com o módulo 8 do PRODIST, estão dentro do percentual de variação adequado. A figura 5 demonstra os resultados gerados na análise.

Figura 5: Resultados obtidos na medição em cada fase

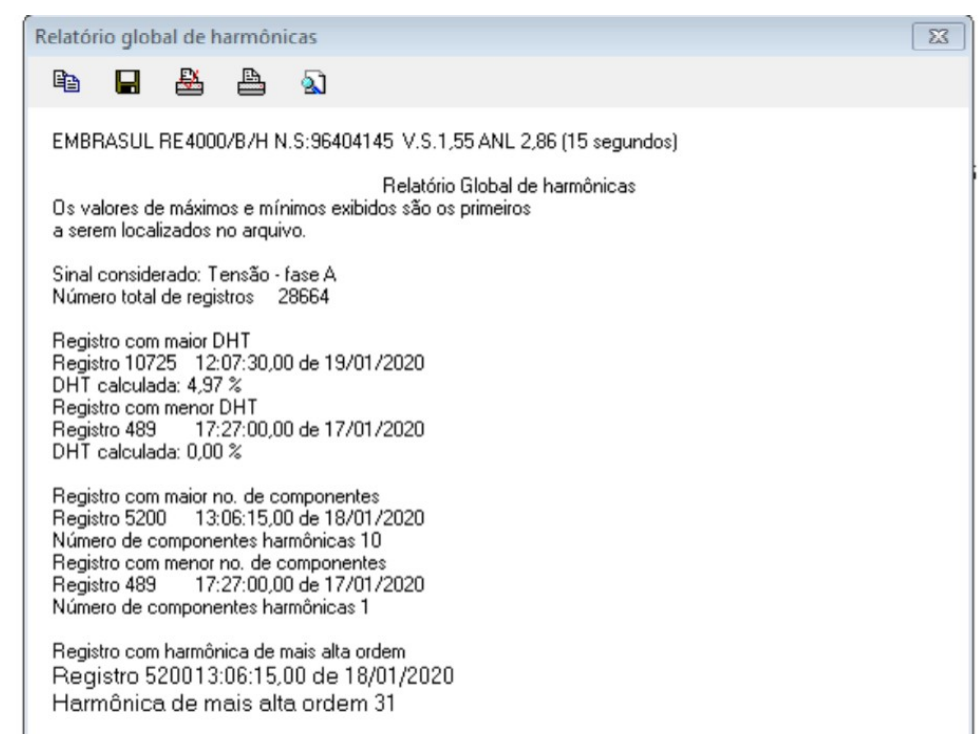


Fonte: Relatório de Qualidade da Energia executado em campo (2020)

Figura 6: Resultados obtidos na medição do fator de potência

EMBRASUL RE4000/B/H N.S:98404145 V.S. 1,55 ANL 2,86 (15 sequndos)

SEX 17/01/2020, 15:45:15,00 até QUA. 22/01/2020, 09:55:00,00

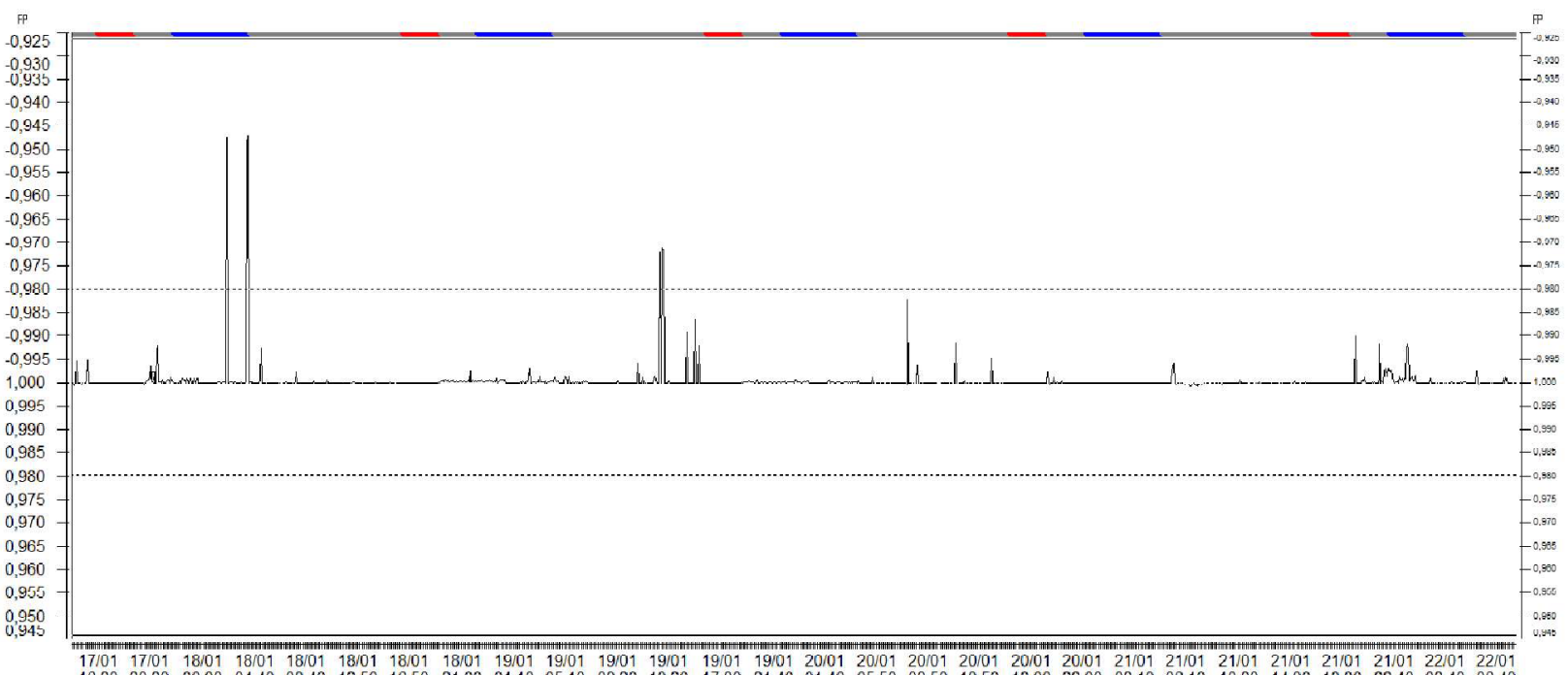

Fonte: Relatório de Qualidade da Energia executado em campo (2020)

O gráfico da Figura 7 apresenta os valores de tensão de linha e tensão de fase amostrados no período. Uma melhor verificação dos dados pode ser feita através das Figura 8 e Figura 9. Os índices DRC e DRP dizem respeito à validade das leituras, descartando amostras em que ocorrem distúrbios elétricos capazes de invalidar a leitura. As violações observadas estão dentro dos limites estabelecidos por resolução, validando as leituras analisadas.

Observa-se que os valores de tensão por fase estão entre um mínimo de $97 \%$ e um máximo de $104 \%$ da tensão nominal, dentro da variação de $5 \%$ previstos por normas.

Figura 7: Valores de tensão de linha e tensão de fase 
EMBRASUL RE4000/B/H N.S:96404145 V.S.1,55 ANL 2.86 (15 segundos)

SEX 17/01/2020, 15:25:15,00 até QUI 23/01/2020, 07:29:30,00

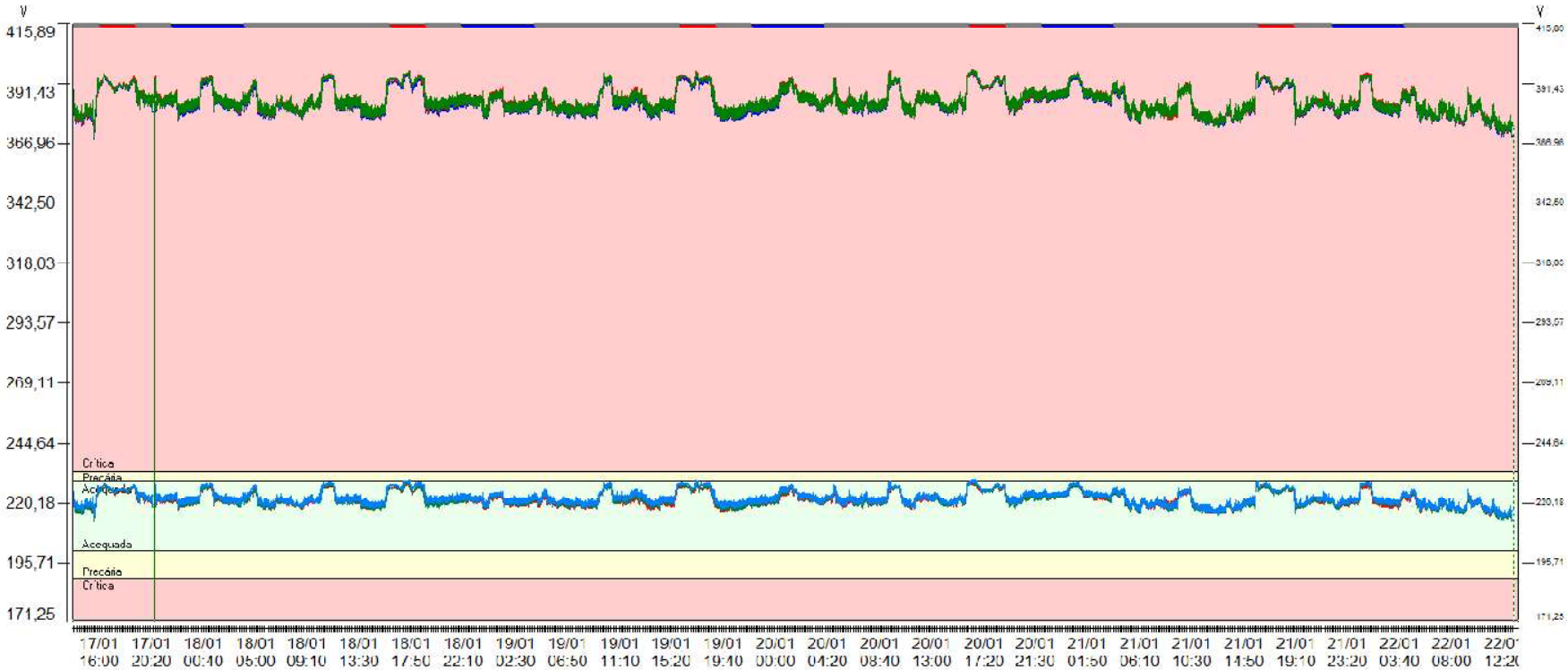

Fonte: Relatório de Qualidade da Energia executado em campo (2020)

Figura 8: Dados da leitura de tensão por fase

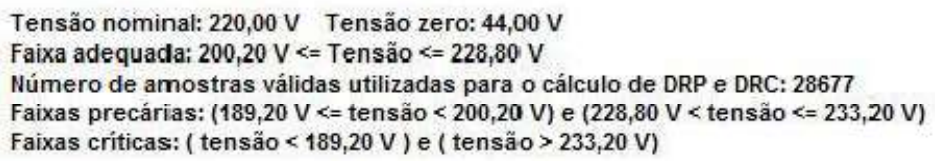

28677

(4) $0,01 \%$

(D) $0,00 \%$

(28673) $99,99 \%$

(28613) $99,79 \%$

(0) $0,00 \%$

(9) $0,03 \%$

(9) $0,03 \%$ $0,03 \%$

(51) $0,18 \%$

(D) $0,00 \%$

(51) $0,18 \%$

$0,18 \%$

$221,43 \mathrm{~V}$

$229,34 \mathrm{~V}$

$126,84 \mathrm{~V}$

Há violações dentro dos limites estabelecidos pela Resolução 505
Pior fase: Va
DRC: $0,18 \%$ limite(0.5\%) DRP: $0,54 \%$ limite( $(3.0 \%)$

iolaçóes dentro dos limites estabelecidos pela Resolução 505
Pior fase: Va
DRC: $0,18 \%$ limite(0.5\%) DRP: $0,54 \%$ limite(3.0\%)

iolaçóes dentro dos limites estabelecidos pela Resolução 505
Pior fase: Va
DRC: $0,18 \%$ limite(0.5\%) DRP: $0,54 \%$ limite(3.0\%)

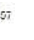

$\begin{array}{rr}\text { Ub } & \text { Uc } \\ 28677 & 28677 \\ (4) 0,01 \% & (4) 0,01 \% \\ (0) 0,00 \% & (0) 0,00 \% \\ 28673) 99,99 \% & (28673) 99,99 \% \\ 28640) 99,88 \% & (28519) 99,46 \% \\ (0) 0,00 \% & (0) 0,00 \% \\ (33) 0,12 \% & (154) 0,54 \% \\ \text { (33) } 0,12 \% & (154) 0,54 \% \\ 0,12 \% & 0,54 \% \\ (0) 0,00 \% & (0) 0,00 \% \\ \text { (0) } 0,00 \% & (0) 0,00 \% \\ \text { (0) } 0,00 \% & (0) 0,00 \% \\ 0,00 \% & 0,00 \% \\ 221,70 \mathrm{~V} & 222,31 \mathrm{~V} \\ 229,42 \mathrm{~V} & 229,59 \mathrm{~V} \\ 212,67 \mathrm{~V} & 213,87 \mathrm{~V}\end{array}$

Fonte: Relatório de Qualidade da Energia executado em campo (2020)

Figura 9: Variação de tensão em percentual 


$\begin{array}{lrrrr}\text { Faixas (\%)(valor) } & \text { Ua }(\%) & \text { Ub }(\%) & \text { Uc }(\%) \\ <80 \%(176,00 \mathrm{~V}, & 0,18 & 0,00 & 0,00 \\ 80 \%(176,00 \mathrm{~V}) & 0,00 & 0,00 & 0,00 \\ 81 \%(178,20 \mathrm{~V}) & 0,00 & 0,00 & 0,00 \\ 82 \%(180,40 \mathrm{~V}) & 0,00 & 0,00 & 0,00 \\ 83 \%(182,60 \mathrm{~V}) & 0,00 & 0,00 & 0,00 \\ 84 \%(184,80 \mathrm{~V}) & 0,00 & 0,00 & 0,00 \\ 85 \%(187,00 \mathrm{~V}) & 0,00 & 0,00 & 0,00 \\ 86 \%(189,20 \mathrm{~V}) & 0,00 & 0,00 & 0,00 \\ 87 \%(191,40 \mathrm{~V}) & 0,00 & 0,00 & 0,00 \\ 88 \%(193,60 \mathrm{~V}) & 0,00 & 0,00 & 0,00 \\ 89 \%(195,80 \mathrm{~V}) & 0,00 & 0,00 & 0,00 \\ 90 \%(198,00 \mathrm{~V}) & 0,00 & 0,00 & 0,00 \\ 91 \%(200,20 \mathrm{~V}) & 0,00 & 0,00 & 0,00 \\ 92 \%(202,40 \mathrm{~V}) & 0,00 & 0,00 & 0,00 \\ 93 \%(204,60 \mathrm{~V}) & 0,00 & 0,00 & 0,00 \\ 94 \%(206,80 \mathrm{~V}) & 0,00 & 0,00 & 0,00 \\ 95 \%(209,00 \mathrm{~V}) & 0,00 & 0,00 & 0,00 \\ 96 \%(211,20 \mathrm{~V}) & 0,00 & 0,01 & 0,00 \\ 97 \%(213,40 \mathrm{~V}) & 0,98 & 1,28 & 0,59 \\ 98 \%(215,60 \mathrm{~V}) & 5,25 & 5,42 & 3,53 \\ 99 \%(217,80 \mathrm{~V}) & 27,00 & 24,71 & 18,00\end{array}$

$\begin{array}{lrrr}\text { Faixas (\%)(valor) } & \text { Ua }(\%) & \text { Ub (\%) } & \text { Uc (\%) } \\ 100 \%(220,00 \mathrm{~V}) & 31,35 & 31,85 & 34,35 \\ 101 \%(222,20 \mathrm{~V}) & 14,19 & 15,17 & 19,17 \\ 102 \%(224,40 \mathrm{~V}) & 13,81 & 12,83 & 10,79 \\ 103 \%(226,60 \mathrm{~V}) & 7,21 & 8,61 & 13,03 \\ 104 \%(228,80 \mathrm{~V}) & 0,03 & 0,12 & 0,54 \\ 105 \%(231,00 \mathrm{~V}) & 0,00 & 0,00 & 0,00 \\ 106 \%(233,20 \mathrm{~V}) & 0,00 & 0,00 & 0,00 \\ 107 \%(235,40 \mathrm{~V}) & 0,00 & 0,00 & 0,00 \\ 108 \%(237,60 \mathrm{~V}) & 0,00 & 0,00 & 0,00 \\ 109 \%(239,80 \mathrm{~V}) & 0,00 & 0,00 & 0,00 \\ 110 \%(242,00 \mathrm{~V}) & 0,00 & 0,00 & 0,00 \\ 111 \%(244,20 \mathrm{~V}) & 0,00 & 0,00 & 0,00 \\ 112 \%(246,40 \mathrm{~V}) & 0,00 & 0,00 & 0,00 \\ 113 \%(248,60 \mathrm{~V}) & 0,00 & 0,00 & 0,00 \\ 114 \%(250,80 \mathrm{~V}) & 0,00 & 0,00 & 0,00 \\ 115 \%(253,00 \mathrm{~V}) & 0,00 & 0,00 & 0,00 \\ 116 \%(255,20 \mathrm{~V}) & 0,00 & 0,00 & 0,00 \\ 117 \%(257,40 \mathrm{~V}) & 0,00 & 0,00 & 0,00 \\ 118 \%(259,60 \mathrm{~V}) & 0,00 & 0,00 & 0,00 \\ 119 \%(261,80 \mathrm{~V}) & 0,00 & 0,00 & 0,00 \\ >120 \%(264,00 \mathrm{~V} & 0,00 & 0,00 & 0,00\end{array}$

Relatório de Qualidade da Energia executado em campo (2020)

A avaliação do desequilíbrio entre as tensões é mostrada pela Figura 9, e conforme índices NEMA e IEC, respectivamente $0,251 \%$ e $0,282 \%$. O valor do FD\% (fator de desequilíbrio) registrado está dentro do limite referencial de $2 \%$ do Módulo 8 do Prodist.

Figura 10: Desequilíbrio entre as tensões

EMBRASUL RE1000/B/H N.S:96101115 V.S.1,55 ANL 2,86 (15 segundos)

SEX 17/01/2020 15:33:45.00 até QUI 23/01/2020 07:29:30.00

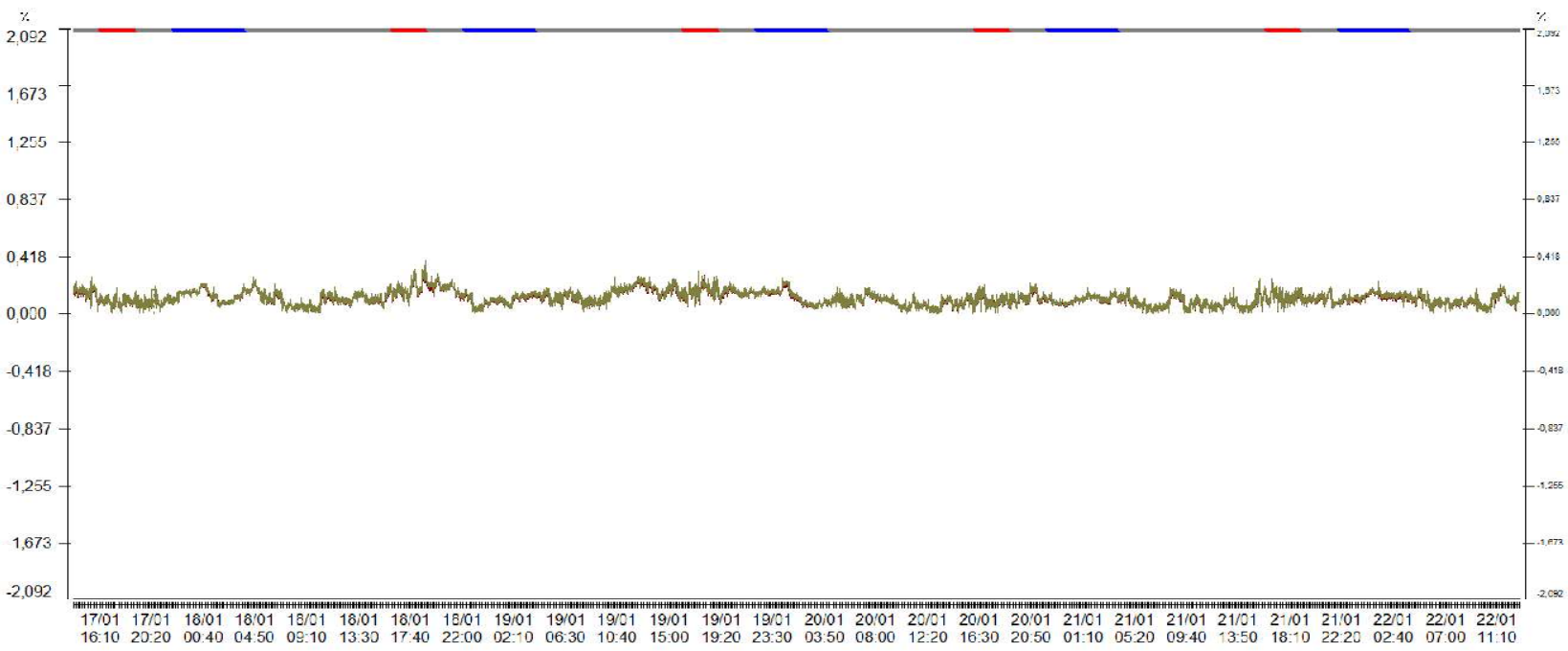

Fonte: Relatório de Qualidade da Energia executado em campo (2020)

\section{CONCLUSÃO}

Os indicadores de qualidade de energia analisados no ponto de interesse se mostraram satisfatórios e em conformidade com as normas e recomendações específicas. É importante salientar 
que os valores aferidos sofrem interferência direta não apenas das cargas e modo de operação, mas da qualidade do fornecimento da concessionária. Ou seja, se os níveis de tensão no ponto de entrega sofrem alteração, os valores de tensão na instalação também são alterados. Ainda, se a alimentação da concessionária estiver poluída de frequências harmônicas, estas serão somadas às geradas internamente. No período da medição não foram verificados afundamentos de tensão fora dos limites aceitáveis de 5\% para mais ou para menos previstos por norma.

Um ponto à ressaltar é que no circuito onde o autotrafo regulador foi intalado, a qualidade da energia melhorou. Isso leva ao questionamento se os problemas que podem estar ocorrendo em alguns equipamentos podem ser devido ao fato da faixa de variação de tensão aceitável dos mesmos serem mais estreitas que as admitidas das normas brasileiras

\section{REFERÊNCIAS BIBLIOGRÁFICAS}

Kagan, N; Robba, E. J; Schmidt, H. P; Estimação de indicadores de qualidade da energia elétrica. São Paulo: Blucher, 2009.

ANEEL - Agência Nacional de Energia Elétrica: Procedimento de Distribuição de Energia

Elétrica no Sistema Elétrico Nacional - PRODIST. Módulo 8 - Qualidade da Energia Elétrica, 2008.

Cubi energia: O que é fator de potência? Disponível em: https://www.cubienergia.com/o-que-efator-de-potencia/. Acesso em 08 de abril de 2020

CAP TECH: Power Quality Solutions. What is Power Quality? https://captech.com.au/what-ispower-quality/. Acesso em 22 de abril de 2020. 